\title{
Adsorption of Methylene Blue Using Activated Carbon Made from Watermelon Rinds
}

\author{
A. Garba ${ }^{1}$, A. Tahir ${ }^{1}$, A. K. Yusuf ${ }^{1 *}$ \\ ${ }^{1}$ Department of Chemistry, College of Natural and Applied Sciences, \\ Al-Qalam University Katsina, Katsina, 820102, NIGERIA \\ *Corresponding Author
}

DOI: https://doi.org/10.30880/jsmpm.2021.01.01.005

Received 15 October 2021; Accepted 01 December 2021; Available online 08 December 2021

\begin{abstract}
This work reports the possibility of using sustainable waste from watermelon rinds as a potential candidate for the removal of Methylene Blue (MB) from aqueous solution in batch mode. The adsorbent was characterized by FTIR and SEM where the FTIR analysis shows peaks at $3370 \mathrm{~cm}^{-1}$ that corresponds to $-\mathrm{OH}$ stretching vibration for lignin, pectin and cellulose, at $1728 \mathrm{~cm}^{-1}$ corresponds to $-\mathrm{C}=\mathrm{O}$ stretching of esters, carboxylic acids, and as well peak in the range of $1350-1000 \mathrm{~cm}^{-1}$ which indicates stretching vibration of alcohols and carboxylic acids. The availability of hydroxyl and carboxyl groups enhance high MB uptake at lower $\mathrm{pH}$. The SEM image of raw adsorbent shows no development of pores, but after carbonization the pores were developed due to escape of volatile groups during carbonization and activation process. Adsorption studies using batch mode were performed by varying adsorption parameters such as adsorbent dosage, contact time, $\mathrm{pH}$ of the solution and initial dye concentration. The maximum capacity of the adsorbent was found to be $0.4 \mathrm{~g}$ dosage, $\mathrm{pH} 4,20 \mathrm{mg} / \mathrm{L} \mathrm{of}$ initial MB concentration and 60 minutes contact time that removes maximum of $197.5 \mathrm{mg} \mathrm{g}^{-1}$. The results indicated that watermelon rind is a successful agricultural waste that could be utilized for sustainable removal of cationic dyes in aqueous solutions.
\end{abstract}

Keywords: Activated carbon, methyl blue, watermelon rinds, adsorption, batch mode

\section{Introduction}

Currently, one of the many issues disturbing people is lack of portable water for drinking and sanitation purposes. This problem is usually caused by human activities through the use of large organic or mineral pollutants from industrial, urban or Agricultural practices. The textile industry is one of the major pollutants contributors through discharge of large amounts of wastewater loaded with dyes and pigments into the environment [1]. Dyes could be categorized as either natural or synthetic organic compounds which provides colouration on other substances. Their classification is usually based on their chemical constituents such as azo dyes, nitrates, nitrous, indigoid, xanthenes, anthraquinones, and phthalocyanines. They can also be classified according to their ionic charges such as acidic, basic, direct, vat, and biting dyes. These dyes are widely utilized in textile industries due to their ease of production, chemical stability and display of variety of colours.

On the other hand, their indiscriminate discharge into the environment causes secondary problems such as emission into the atmosphere, high energy consumption, deposition of solid waste, and generation of bad odour [2]. These dyes were consumed in several industries such as rubber, plastic, paper, leather, food, pharmaceutical, cosmetic and textile industries. However, poorly treated effluents discharged from textile industries contain dyes which exhibit high Chemical Oxygen Demand (COD), high Biological Oxygen Demand (BOD), resistance to degradation, carcinogenic, allergic reaction, dermatitis, rhinitis, occupational asthma, mutagenic, high amount of dissolved solids, and are visibly coloured [3]. The maximum allowed concentration for COD in wastewater is $4.0 \mathrm{mg} / \mathrm{L}$, while BOD concentration should not exceed $300 \mathrm{mg} / \mathrm{L}$ in accordance with the United State Public Health (USPH) set standards [4]. 
However, due the carcinogenic effects, high chemical and thermal stability, and resistance to degradation by heat, light, and natural oxidants, there is urgent need for their removal from wastewater [5].

Various technologies have been utilized for the removal of dye stuffs from wastewater such as electrocoagulation, coagulation-flocculation, membrane separation, ion-exchange, biological treatment, advanced oxidation, and adsorption using activated carbon. However, all the above-mentioned removal technologies (excluding adsorption) have disadvantages such as high consumption of energy, use of expensive equipment or high operational cost, incomplete removal of target pollutants, generation of toxic byproducts, and issues concerning disposal of such byproducts [6-8]. Adsorption techniques for dyes using agricultural materials was much preferred over all the other methods of removal. This could be attributed to the advantages of the process which it is effective, cheap, flexible, simplicity of design, insensitivity to toxic pollutants, and the procedure is easy to undertake [9]. But, the cost of producing commercial activated carbons is high which made scientist to look for alternative precursor materials that are cheaper, sustainable, and easy to regenerate $[10,11]$.

The aim of this paper is to explore the removal capacity of activated carbon prepared from sustainable watermelon rinds for the removal of Methylene blue as a model organic dye.

\section{Materials and Methods}

\subsection{Materials and Reagents}

Sodium hydroxide, sodium chloride, hydrochloric acid and MB were all purchased from Sigma Aldrich chemicals, Germany. All reagents used were of analytical grade and were prepared in a $1000 \mathrm{~mL}$ flask. The flasks were prewashed, rinsed with distilled water and appropriate quantity of the MB was dissolved to prepare $1000 \mathrm{mg} / \mathrm{L}$. The stock solution was then diluted so as to prepare $\mathrm{MB}$ concentrations of $10 \mathrm{mg} / \mathrm{L}, 20 \mathrm{mg} / \mathrm{L}, 30 \mathrm{mg} / \mathrm{L}, 40 \mathrm{mg} / \mathrm{L}$ and $50 \mathrm{mg} / \mathrm{L}$ respectively.

\subsection{Sample Preparation}

Watermelon Rinds (WMR) was obtained from a local seller in layout around katsina metropolis and washed thoroughly to remove sand and other impurities, the rinds were then cut into smaller pieces and air dried until the moisture content were removed, and the WMR was impregnated in a solution of $\mathrm{Zinc}$ Chloride $\left(\mathrm{ZnCl}_{2}\right)$ by soaking for 24 hours. Sufficient quantity was also soaked well with $10 \%$ chloride solution so that the solution gets thoroughly absorbed. The excess chloride solution was poured out and the materials were dried in the air. The dried sample was carbonized at $800^{\circ} \mathrm{C}$ for about 2 hours; the activated WMR was powdered for use [12].

\subsection{Batch Adsorption}

Adsorption of the MB on the adsorbent was determined by batch mode equilibrium method. All the batch mode experiments were carried out in conical flasks $(100 \mathrm{~mL})$ that contains $25 \mathrm{~mL}$ of the adsorbate solutions. The adsorption capacity of the adsorbent was explored by mixing it with $0.2-1.2 \mathrm{~g}$ with $25 \mathrm{~mL}$ of known MB concentrations of 20 $\mathrm{mg} / \mathrm{L}-50 \mathrm{mg} / \mathrm{L}$ between 5 to 75 minutes at room temperature. The mixture was separated by filtration using a filter paper and the percentage removal from the filtrates was determined using UV-vis spectrophotometer. The effect of $\mathrm{pH}$ on adsorption capacity of the adsorbent was determined in the range of $\mathrm{pH} 4-8$ using either $0.1 \mathrm{M} \mathrm{HCl} \mathrm{or} 0.1 \mathrm{M} \mathrm{NaOH}$ to obtain a desired $\mathrm{pH}$ value. All experimental results were obtained in triplicates and average values were reported in this work. The amount of MB adsorbed on the adsorbent was determined by Equation 1, and the percentage removal was deduced using Equation 2 below:

$$
\begin{aligned}
& q_{e}=\left(C_{0}-C_{g}\right) \frac{w}{m} \\
& \% \text { Removal }=\frac{\left(C_{0}-C_{g}\right)}{C_{\mathrm{n}}} \times 100
\end{aligned}
$$

Where $\mathrm{Co}$ and $\mathrm{C}_{\mathrm{f}}$ are the initial and final concentrations in $\mathrm{mg} / \mathrm{L}$, qe $\mathrm{MB}$ uptake in $\mathrm{mg} / \mathrm{g}$ of adsorbent, $\mathrm{v}$ is the volume of solution in $\mathrm{L}$ and $\mathrm{m}$ is the mass of adsorbent used [13].

\section{Results and Discussion}

\subsection{Characterization of the Adsorbent}

The prepared adsorbent from watermelon rinds was characterized with FTIR analysis (ThermoFisher Nicolet iN10 IR) so as to understand the surface function groups that help in binding MB on the adsorbent. From Fig. 1, the FTIR analysis displays peaks at different wavenumbers indicating presence of several groups. The broad peak at $3378 \mathrm{~cm}^{-1}$ confirms $-\mathrm{OH}$ stretching vibrations of lignin, pectin and cellulose, while the one at $2917 \mathrm{~cm}^{-1} \mathrm{corresponds}$ to $\mathrm{CH}$ 
stretching vibrations of methoxy and methyl groups. Peaks were also seen at $1728 \mathrm{~cm}^{-1}$ corresponding to $-\mathrm{C}=\mathrm{O}$ stretching of esters, carboxylic acids, symmetric and asymmetric vibrations of -COOC- (ionic carboxylic groups). The peaks noticed from $1350 \mathrm{~cm}^{-1}$ to $1000 \mathrm{~cm}^{-1}$ corresponds to stretching vibrations of alcohols and carboxylic acids respectively. The FTIR spectrum of the adsorbent shows that hydroxyl and carboxylic groups were highly available and as biopolymers, the groups act as proton donors that help in binding cations.

The surface morphology of the adsorbent was examined with scanning electron microscope (Angstrom AA8000 model). The SEM images of the raw and treated watermelon rinds was shown in Fig. 2. It can be seen that, Fig. $2 \mathrm{a}$. shows the image of the raw adsorbent with very little pores, while Fig. $2 b$. shows that pores were developed due to subsequent carbonization and activation of the precursor material which allow the volatile components to leave, thus creating the pores.

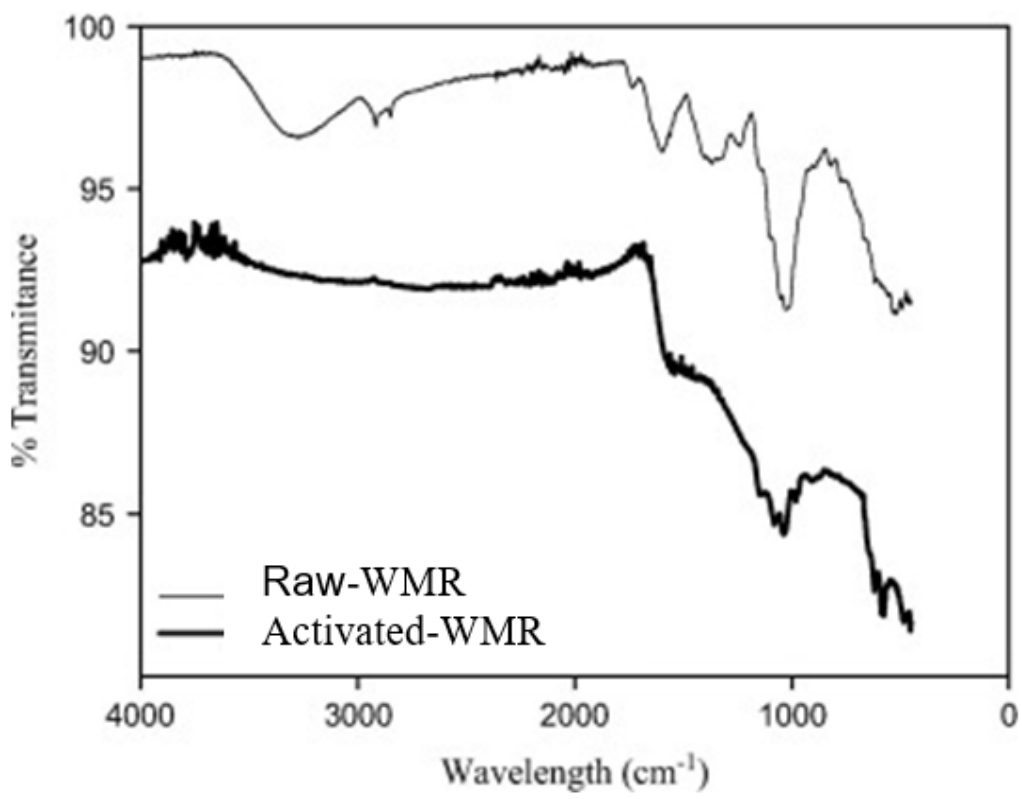

Fig. 1 - FTIR spectra of watermelon rinds
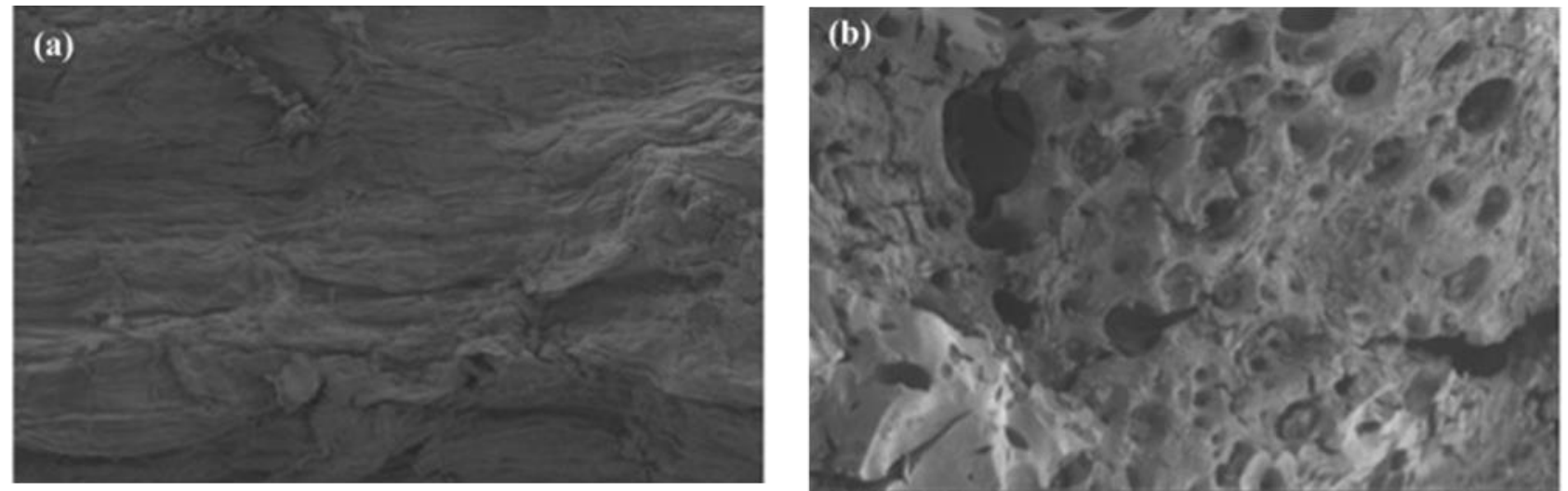

Fig. 2 - (a) SEM image of raw watermelon rind; (b) SEM image of carbonized watermelon rind

\subsection{Effect of Contact Time}

Fig. 3 indicated the effect of contact time on the adsorbent for MB removal. It shows that, the adsorbent started MB uptake until all the adsorption sites were saturated so that any further contact will not increase the removal of the adsorbate from solution. At this instance, the amount of MB desorbing from the adsorbent is the same as that amount adsorbed. Therefore, an equilibrium is established and the time taken to reach this state is regarded as equilibrium time which signifies the maximum adsorption capacity of the adsorbent. The indicated that, the adsorption capacity was reached around 60 minutes, but the reaction was allowed to reach up to 75 minutes for complete adsorption. The maximum adsorption capacity was found to be $197.5 \mathrm{mg} \mathrm{g}^{-1}$. The result agreed with what was reported by Jawad et. al. on the utilization of watermelon rinds for the adsorption of methylene blue [14]. 


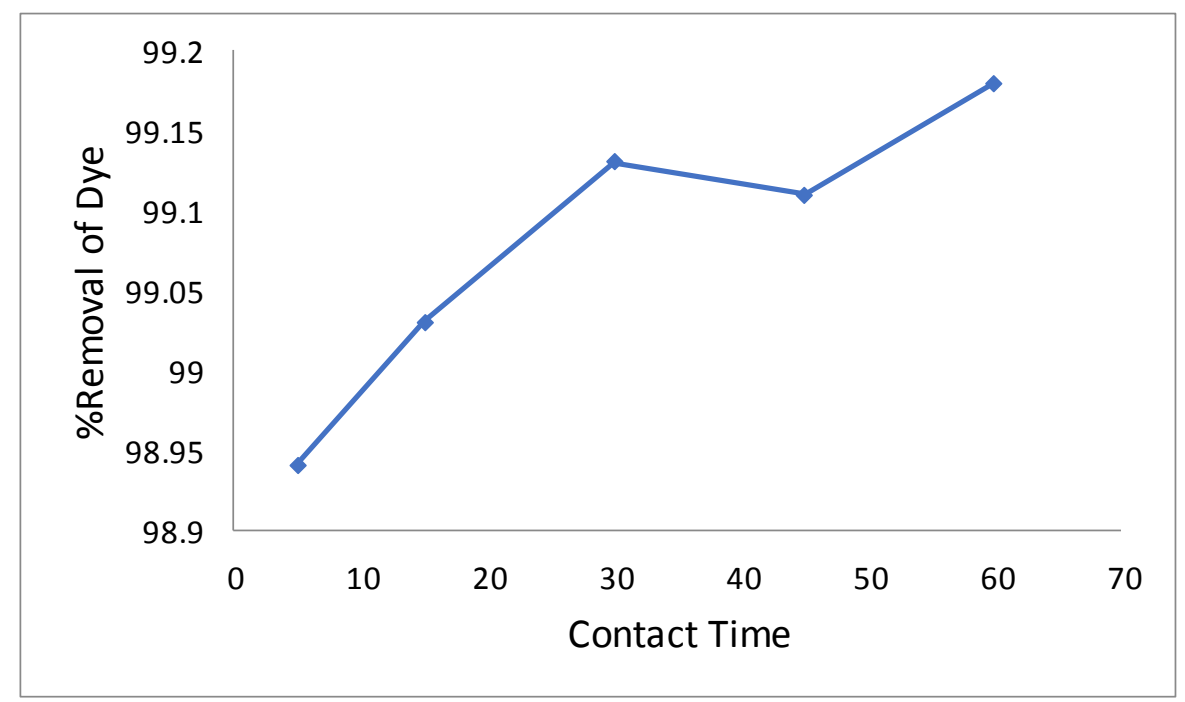

Fig. 3 - Absorbance of dye against contact time

\subsection{Effect of Adsorbent Dosage}

The effect of adsorption doses was studied by agitating $0.2,0.4,0.6,0.8,1.0,1.2$, and $1.4 \mathrm{~g}$ of dye in different sample solutions for same time periods of 75 minutes. From Fig. 4 , it was observed that there is a proportional increase in dye removal with corresponding increase in adsorbent dose. This is due to increase in adsorption sites as the dosage increases which results in high removal of the dye. The optimum dosage was found to be $0.4 \mathrm{~g}$.

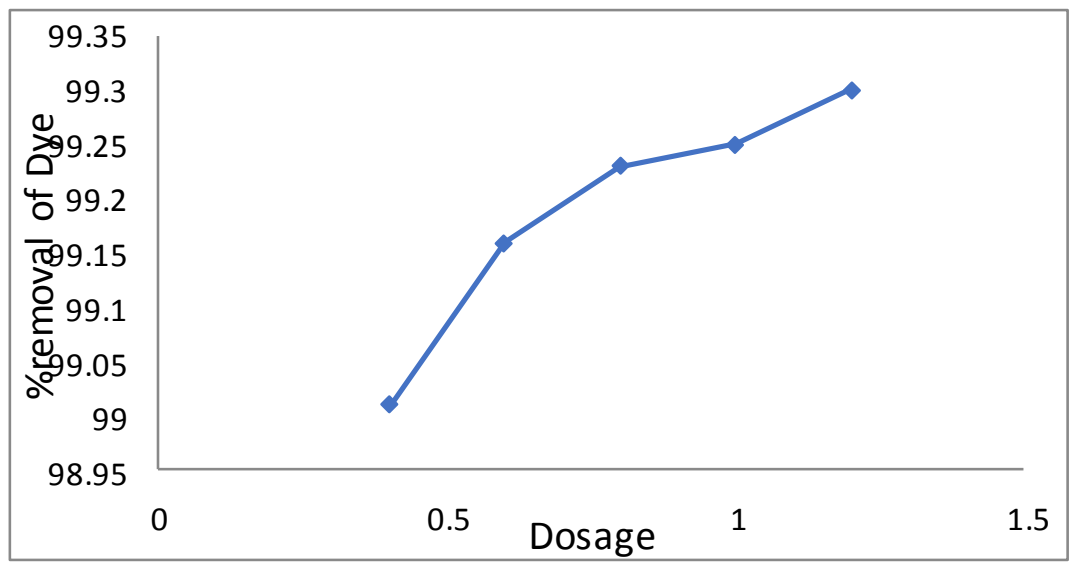

Fig. 4 - Absorbance of dye against dosage

\subsection{Effect of Initial MB Concentration}

The effect of MB concentration was studied by varying the initial adsorbate concentrations between the range of $10-50 \mathrm{mg} / \mathrm{L}$ at room temperature using $0.4 \mathrm{~g}$ of the adsorbent throughout and keeping the initial $\mathrm{pH}$ at constant value of 4. The MB uptake is higher at lower concentrations which keeps decreasing with an increase in the initial MB concentration. It could be deduced that the percentage removal was reducing due presence of more MB ions that were readily available for adsorption as the initial concentrations of MB is increased. Therefore, this means that adsorption is highly dependent initial concentration of the adsorbate.

\subsection{Effect of pH}

Fig. 6 shows that maximum uptake of dye was observed at $\mathrm{pH} 4$ using initial dye concentration of $20 \mathrm{mg} / \mathrm{L}$ and adsorbent concentration of $0.4 \mathrm{~g}$. At range of $\mathrm{pH} 2-7$, the percentage removal increases sharply, while at higher $\mathrm{pH} 8-$ 10 there is a little decrease. The $\mathrm{pH}$ of an adsorbate could affect adsorption in relation with the surface groups on the adsorbent. The carbonized watermelon rinds contain high amount of carboxyl and hydroxyl groups which were negatively charged, while the adsorbate is a cationic dye. Thus, at lower $\mathrm{pH}$ of $2-4$, the adsorbent surface is negatively charged and the $\mathrm{MB}$ is positively charged which enhance adsorption process thereby making the process sharp. On the 
other hand, when adsorption is carried out at higher $\mathrm{pH}$ of $8-10$, there is large amount of hydroxyl ions from the solution which hinders the process thereby slowing down the MB uptake.

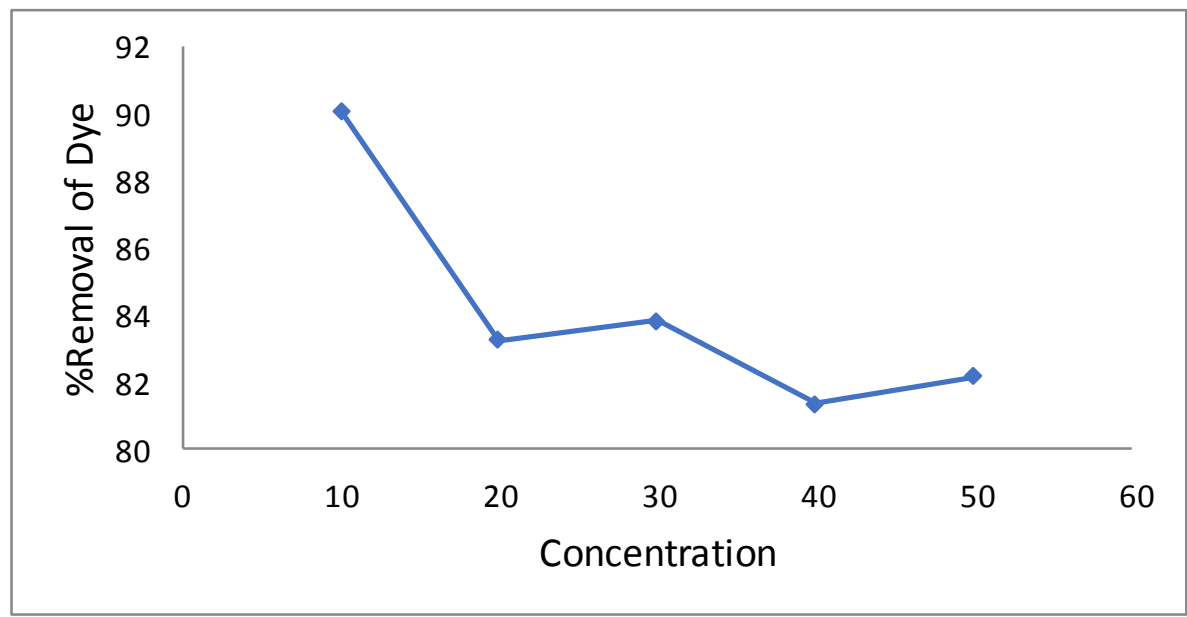

Fig. 5 - Absorbance of dye against initial MB concentration

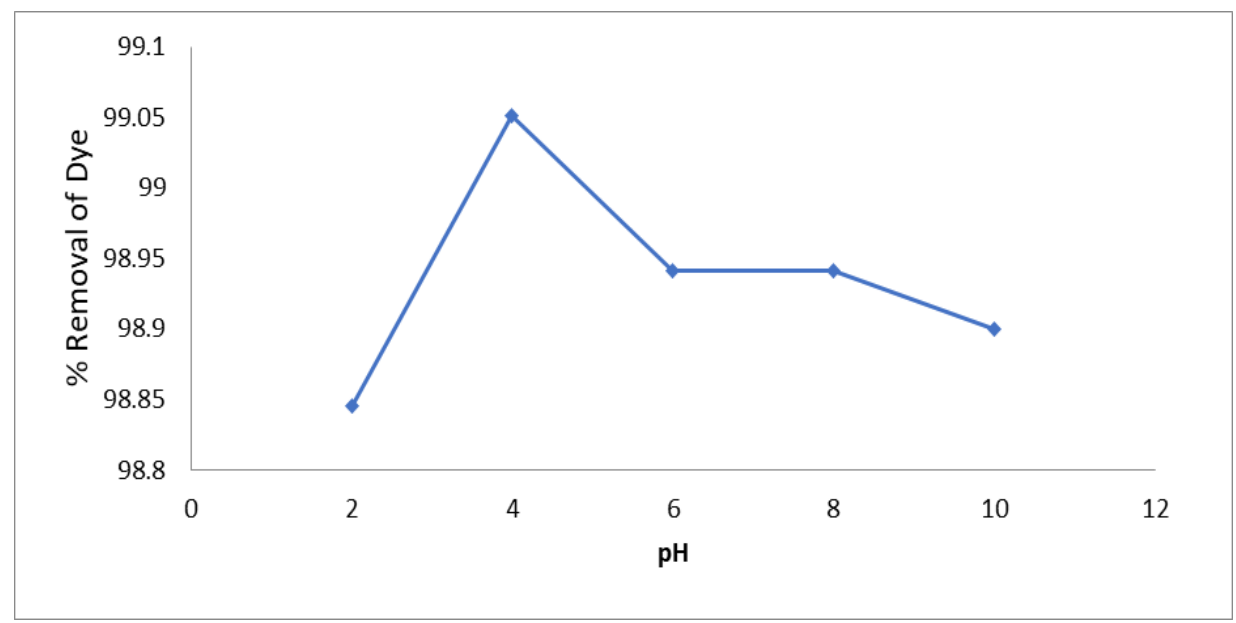

Fig. 6 - Absorbance of dye against pH

\section{Conclusion}

Watermelon rinds which were an agricultural waste was carbonized and activated for the removal of MB from aqueous solution by batch adsorption mode. The results indicated that all the adsorption parameters have influence on the adsorption process. The results also shows that watermelon rind is a successful agricultural waste that could be utilized for sustainable removal of cationic dyes in aqueous solutions.

\section{Acknowledgement}

The authors would like to thank Al-Qalam University Katsina for the financial support rendered during the course of conducting this research.

\section{References}

[1] Osagie, C., Othmani, A., Ghosh, S., Malloum, A., KashitarashEsfahani, Z., \& Ahmadi, S. (2021). Dyes adsorption from aqueous media through the nanotechnology: A review. Journal of Materials Research and Technology.

[2] Berradi, M., et al., Textile finishing dyes and their impact on aquatic environs. Heliyon, 2019. 5(11).

[3] Katheresan, V., Kansedo, J., \& Lau, S. Y. (2018). Efficiency of various recent wastewater dye removal methods: A review. Journal of environmental chemical engineering, 6(4), 4676-4697.

[4] Katheresan, V., Kansedo, J., \& Lau, S. Y. (2018). Efficiency of various recent wastewater dye removal methods: A review. Journal of environmental chemical engineering, 6(4), 4676-4697. 1. 
[5] Saini, R. D. (2017). Textile organic dyes: polluting effects and elimination methods from textile waste water. Int J Chem Eng Res, 9(1), 121-136.

[6] Homaeigohar, S. (2020). The nanosized dye adsorbents for water treatment. Nanomaterials, 10(2), 295.

[7] Othmani, A., Kesraoui, A., \& Seffen, M. (2017). The alternating and direct current effect on the elimination of cationic and anionic dye from aqueous solutions by electrocoagulation and coagulation flocculation. EuroMediterranean Journal for Environmental Integration, 2(1), 6.

[8] Soonmi, H. (2018). Removal of dye by adsorption onto activated carbons. Eurasian Journal of Analytical Chemistry, 13(4), 332-338.

[9] Garba, A., Basri, H., Nasri, N. S., Siddiq, U. H., \& Rahman, A. R. A. (2017). Modeling of lead (II) adsorption on sodium hydroxide treated rice husk: Fixed-bed studies. Malaysian Journal of Fundamental and Applied Sciences, 13(4), 803-806.

[10] Ahmad, A., Mohd-Setapar, S. H., Chuong, C. S., Khatoon, A., Wani, W. A., Kumar, R., \& Rafatullah, M. (2015). Recent advances in new generation dye removal technologies: novel search for approaches to reprocess wastewater. RSC advances, 5(39), 30801-30818.

[11] Uddin, M. T., Islam, M. A., Mahmud, S., \& Rukanuzzaman, M. (2009). Adsorptive removal of methylene blue by tea waste. Journal of Hazardous Materials, 164(1), 53-60.

[12] Garba, A., H. Basri, and N.S. Nasri, Adsorption of Bisphenol A from Aqueous Solution on Ammonia Modified Carbon: Fixed-Bed Studies. Advanced Science Letters, 2018. 24(5): p. 3573-3578.

[13] Abbas, M. (2021). Modeling of adsorption isotherms of heavy metals onto Apricot stone activated carbon: twoparameter models and equations allowing determination of thermodynamic parameters. Materials Today: Proceedings, 43, 3359-3364.

[14] Jawad, A. H., Ngoh, Y. S., \& Radzun, K. A. (2018). Utilization of watermelon (Citrullus lanatus) rinds as a natural low-cost biosorbent for adsorption of methylene blue: kinetic, equilibrium and thermodynamic studies. Journal of Taibah University for Science, 12(4), 371-381. 